Published in: Journal of Cleaner Production 263 (2020) 121337.

https://doi.org/10.1016/j.jclepro.2020.121337

\title{
Generating electricity with urban green infrastructure microbial fuel cells
}

Theodore Endreny ${ }^{1}$, Claudio Avignone-Rossa ${ }^{3}$, Rosa Anna Nastro ${ }^{2,3 *}$

${ }^{1}$ Department of Environmental Resources Engineering, SUNY ESF, 1 Forestry Drive, Syracuse, New York 13210 , USA. te@esf.edu

${ }^{2}$ Department of Engineering, University of Naples Parthenope, 80134 Naples, Italy.

${ }^{3}$ Department of Microbial Sciences, University of Surrey. Guildford, GU2 7XH, United Kingdom. e-mail: c.avignonerossa@surrey.ac.uk

Corresponding Author e-mail: r.nastro@uniparthenope.it

\begin{abstract}
Urban areas can modify their green infrastructure to include microbial fuel cells and generate electricity to help address energy security. Naturally occurring electroactive bacteria utilize plant compounds and organic pollutants as electron donors. Water is cleaned, electricity is generated, and additional ecological services are provided.
\end{abstract}

Globally, urban areas are struggling to satisfy increasing energy demands, with low-income countries most vulnerable to the environmental, economic, and social costs of energy shortages. Therefore, it is essential to exploit all possible sources of energy. A potential source of energy is the urban green infrastructure (UGI). These systems consist of soil and plants for the capture and processing of urban stormwater, and can include urban forests that use infiltration and evaporation to reduce pollution, erosion, and flooding. UGIs may also contribute to alleviate energy demands by generating electricity through the application of Microbial Fuel Cell (MFC) technology. These are bio-electrochemical systems (BESs) that utilize the biological capacity of microorganisms (single species or microbial communities) to strip electrons from organic carbon sources and catalyze electrochemical reactions, with concomitant production of electricity.

The introduction of MFCs to the urban ecosystem via green infrastructure can lead to new electricity sources, de-pollution of water and soil, and delivering critical ecosystem services such as further 
water purification, cooling air, and filtering air pollution. A most basic Green Infrastructure-MFC (GI-MFC) could resemble a common laboratory MFC system for soil bioremediation, opportunely integrated with plants. Integration of plant + remediation MFCs could result in an improved ratio of carbon to nitrogen to phosphorus $(\mathrm{C}: \mathrm{N}: \mathrm{P})$ for the microbial community. Therefore, GI-MFCs in polluted soils with plants could provide a higher power production in comparison to remediation MFCs without plants. Separately, plant MFCs could be utilized in areas with greater sunlight exposure and fewer pollutants, while systems with less sunlight but with organic pollution could use remediation MFCs. However, why MFCs? These systems can withstand anaerobic, acidic, and saline soils common to urban areas, and still generate good power density and deliver useful current. In anaerobic conditions, which are typically saturated with water, alternative electron acceptors (e.g. nitrate and hydrocarbon pollutants) can be involved in electrogenesis. MFCs have been demonstrated to remediate hydrocarbons across a range of saturation levels, from contaminated waters, to groundwater, to sediment systems. In marine soils, with varying salinity and saturation, anoxic cathodes achieved promising efficiencies in energy production and in the remediation of pollutants. In green infrastructure designs, mixed configurations of MFCs could be established. The designer has the opportunity to mix multiple MFC systems, using aerobic cathodes for relatively dry areas and conditions, and anoxic cathodes for relatively saturated areas and conditions. Even in aerobic conditions, the MFC needs some capillary water to maximize production of electricity and remediation of organic pollutants.

In an urban environment, a membraneless, multi-electrode system with carbon-based materials as electrodes would be a cheap and effective solution to reduce both soil and water pollution while recovering energy (Fig.1). In order to improve the electric output, GI-MFCs can be arranged in stacks by connecting them in parallel and in series according to the "scaling-up through plurality" approach, that has led to significant increase in both power and current production in MFCs applied to wastewater treatment. The expected results would be a faster degradation of organic compounds (including pollutants) with simultaneous power production. A preliminary testing on dry soil (4.0 De 
Martonne index) polluted by hydrocarbons and analyzed after 4 weeks for its content in Polycyclic Aromatic Hydrocarbons (PAHs) showed, for 16 PAHs listed among the priority pollutants, a 34\% reduction in PAHs concentration when soil was treated in MFCs, whereas the decrease observed in control samples (untreated soil) was less than 5\%. Our results are far more remarkable if we consider GI-MFCs showed complete compatibility with plant growth. Due to water scarcity, MFCs sometimes experienced voltage reversal and low power output, yet remaining able to degrade PAH. With increasing soil water content, GI-MFC power performance is expected to be in THE range of 0.01 to $1 \mathrm{~W} / \mathrm{m}^{2}$ and of 0.001 to $1 \mathrm{~A} / \mathrm{m}^{2}$, according to cathode configuration, degree of soil saturation and carbon source, e.g., decaying vegetation or degrading pollutants. In summary, GI-MFC performance is expected to be similar to Constructed Wetlands-, plant- and bioremediation-MFCs.

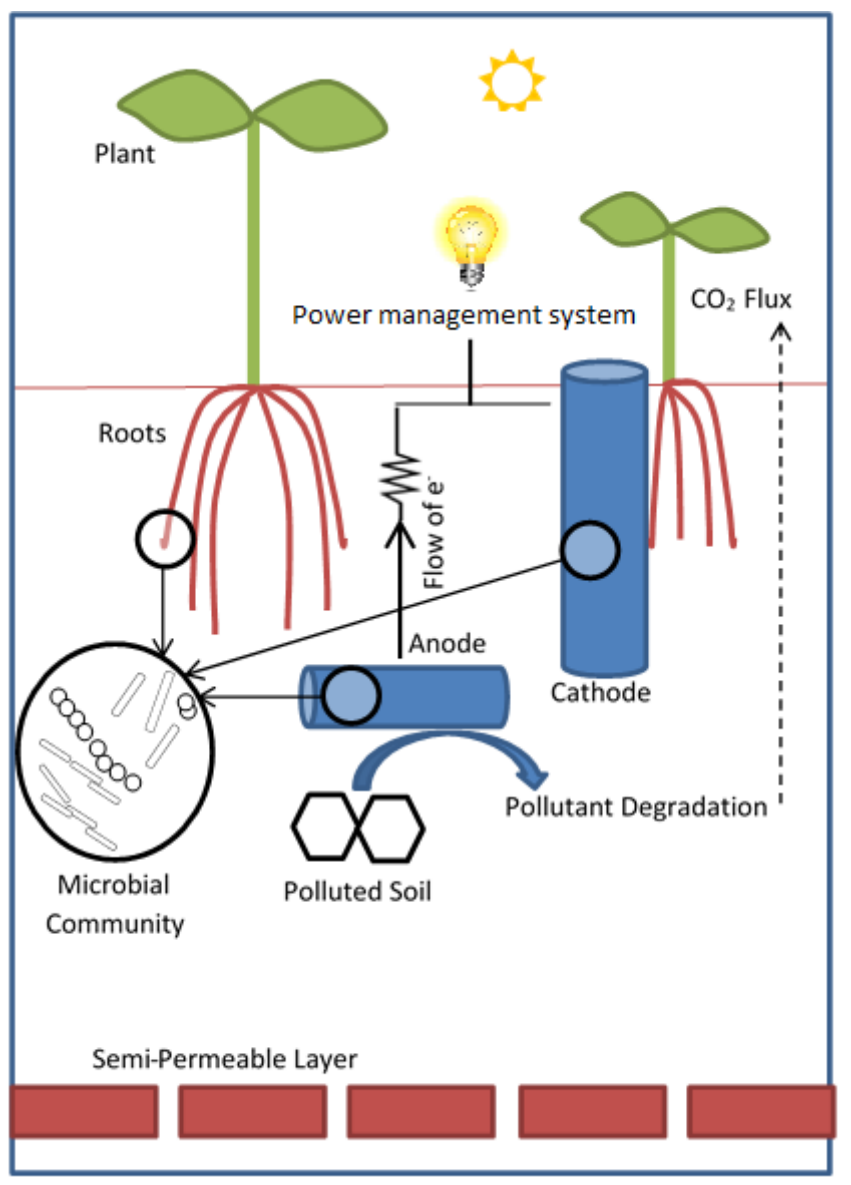

Figure 1: GI-MFCs main scheme.

The GI-MFC would likely be designed to handle fluctuations in water and pollutant conditions, perhaps incorporating spatial and temporal modifications of the MFC system to address changing 
conditions. As an example, for an aerobic GI-MFC system a regular supply of water to the porous soil media could be delivered from slow distribution of stormwater basins or graywater from nearby buildings and facilities. GI-MFC technology has the potential to contribute valuable services for our urban communities, and additional research can likely improve design options for use in optimizing urban sustainability and delivery of ecosystem services to improve human wellbeing. The additional research needed to combine microbial fuel cell and green infrastructure systems will likely emerge from the significant progress being generated by research in these relatively new technologies.

Declaration of competing interest. The authors declare that they have no known competing financial interests or personal relationships that could have appeared to influence the work reported in this paper. 